\title{
Analysis of Glycemic Control of a Pharmacist-Led Medication Management Program in Patients with Type 2 Diabetes
}

\author{
John J. Ko, PharmD, MS; Jackie Lu, PharmD, MS; Karen Rascati, PhD; Eileen M. Stock, PhD; \\ Joyce Juan, PharmD; Kangho Suh, PharmD, MS; Yoona Kim, PharmD; Patricia A. Tabor, PharmD; \\ and Paul J. Godley, PharmD
}

\begin{abstract}
BACKGROUND: An integrated health care system with its own regional health plan located in Texas implemented a pharmacist-led diabetes medication management program (MMP) to treat type 2 diabetic patients (baseline A1c $>7.5 \%$ ). The MMP formed collaborative practice agreements with the system's physicians to allow ambulatory care pharmacists to modify and adjust diabetic drug regimens when appropriate. Enrolled MMP patients received personalized visits with ambulatory care pharmacists and a copay waiver on diabetes medications.
\end{abstract}

OBJECTIVE: To study the outcomes of an outpatient, pharmacist-led MMP, along with a copay waiver on diabetes drugs, in treating adults with type 2 diabetes mellitus over a 2-year period compared with standard care practice.

METHODS: This retrospective study employed a quasi-experimental design and used medical claims, pharmacy claims, eligibility data, and electronic medical records. Patients aged 18 to 62 years, who were diagnosed with type 2 diabetes mellitus, and had at least 1 diabetes-related pharmacy claim in the year before the MMP, as well as continuous enrollment in the health plan, were included. Patients enrolled in the pharmacist-led MMP for at least 2 years $(n=75)$ were matched to standard care patients $(n=75)$ on age, gender, baseline A1c, insulin use, and physical comorbidity. The primary outcome was the 2-year change in A1c. Secondary outcomes included inpatient costs, outpatient costs, and pharmacy costs from the baseline period (year before enrollment) compared with the follow-up period (second year of enrollment).

RESULTS: After matching MMP patients $(n=75)$ to control patients $(n=75)$, the baseline A1c (9.30 and 9.26), the mean age (53.0 and 53.3, respectiveIy), the Selim Physical Score (3.32 and 3.26, respectively), and the use of insulin (56.0\% and $56.0 \%$, respectively) were similar in both groups. MMP patients had a greater mean reduction in A1c compared with standard care patients $(-1.24$ vs. $-0.59, P=0.009)$ from baseline to after 2 years. After 2 years, the A1c for MMP patients was significantly lower compared with control patients ( 8.06 vs.8.67, respectively, $P=0.014$ ). There was also a difference in A1c after 1 year for MMP patients versus control patients (8.18 and 8.69 , respectively, $P=0.012$ ).

CONCLUSIONS: A pharmacist-led diabetes MMP, combined with a diabetes drug copay waiver, was effective in significantly reducing A1c over a 2-year period for type 2 diabetic patients in this regional health plan.

J Manag Care Spec Pharm. 2016;22(1):32-37

Copyright $\odot 2016$, Academy of Managed Care Pharmacy. All rights reserved.

\section{What is already known about this subject}

Outpatient pharmacist interventions are associated with reductions in Alc and improved clinical outcomes for type 2 diabetics. Copay reduction programs for diabetics have been associated with a modest increase in adherence and higher payer prescription expenditures.

\section{What this study adds}

When compared with a matched control cohort, medication management program patients with pharmacist-led intervention combined with a diabetes copay waiver program had significant reductions in Alc after 1 year and was sustained after 2 years.

Patients who received a structured medication management program and copay waiver for diabetes drugs had lower medical cost spending compared with the matched control cohort after 2 years.

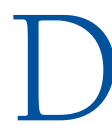
iabetes continues to be a major health concern for individuals and society because of the high physiologic and economic burden brought on by its complications. A recent study found that the national average annual medical cost per case of diabetes was $\$ 6,649$ in 2007 and approached $\$ 10,000$ per case annually when taking the indirect costs of diabetes into account. ${ }^{1}$ In addition, $32 \%$ of hospital admissions in the United States are due to uncontrolled diabetes, which translates to an estimated annual cost of $\$ 2.4$ billion. $^{2}$

A growing body of evidence indicates that diabetes care provided by a pharmacist is effective in improving treatment adherence and lowering patients' glycated hemoglobin Alc (Alc) levels, mainly because of the ability to dedicate time and expertise to providing comprehensive medication review..$^{3-5}$ The Asheville Project and the Diabetes Ten City Challenge provided evidence of the clinical and economic benefits of diabetes care provided by pharmacists. ${ }^{6,7}$ Although the lack of a control group limited generalizability of their findings, these programs demonstrated the feasibility of a pharmacist-led diabetes care model.

Subsequently, several pharmacist-led medication management programs have been developed to improve diabetes control. A review of 16 interventions in inpatient and outpatient settings demonstrated that pharmacist interventions significantly reduced Alc by an average of $0.62 \%$ over controls. ${ }^{4}$ Likewise, in a review of 22 studies, 20 found that pharmacist 
outpatient interventions were associated with Alc changes ranging from an increase of $0.2 \%$ to a decrease of $2.1 \%$ compared with controls. ${ }^{8}$ In addition, interventions that allowed pharmacists to make medication adjustments, as opposed to providing only drug review and disease education, reported greater improvements in Alc. However, most of the study durations were less than 1 year and/or did not have a matched control cohort.

A study examining the effectiveness of a free rural clinic for diabetes patients managed by pharmacists found that patients who received education on diabetes, counseling on lifestyle modifications, and management of diabetes drug therapy had significant reductions in Alc, blood pressure, low-density lipoprotein (LDL), and triglycerides after 24 months. They extrapolated savings of $\$ 1,118$ per patient who had a decrease of $\geq 1 \%$ in Alc based on the findings of Wagner et al. (2001). ${ }^{9}$ A total of 67 out of 95 patients achieved this improvement. ${ }^{9,10}$

In 2013, a study evaluated 12-month clinical markers and long-term cardiovascular risk in type 2 diabetes patients within a health maintenance organization primary care setting who were managed by a team of providers including a pharmacist versus a matched control cohort (physician-only team). ${ }^{11}$ This study found that the patients managed by a team including a pharmacist had significant reductions in Alc after 12 months; were more likely to attain Alc, blood pressure, and LDL goals; and had a significantly lower 10-year risk of coronary heart disease. ${ }^{11}$ This study supports the effectiveness of increasing pharmacists' clinical responsibilities for patients with type 2 diabetes.

Because of the high cost associated with controlling diabetes, health care payers are taking on an increasingly active role in the provision of diabetes management. Previous research found that reducing Alc to target goals $(<7 \%)$ did not lead to immediate short-term cost reductions; however, sustained Alc control was associated with lower costs over a 3-year time period. ${ }^{12}$ When using a Markov model to determine the costeffectiveness of pharmacist care of type 2 diabetes patients in the outpatient setting, pharmacist intervention was a dominant choice (more effective and less expensive) when compared with standard care patients over a 10 -year period. The researchers suggest that pharmacist intervention in the treatment of diabetes patients is more likely to be a cost-effective strategy over longer time horizons of at least 4 to 5 years. ${ }^{13}$

Patient adherence to prescribed diabetes medications often depends on the level of out-of-pocket costs. Pharmacy benefit managers have researched cost reduction strategies in an effort to increase adherence to diabetes medications. In 1 study, copay reductions to the lowest copay tier for all diabetes medications and testing supplies led to a modest increase in adherence and reductions in emergency room visits and hospitalizations, as well as overall health care utilization after 1 year. ${ }^{14}$
Scott \& White Health Plan, an integrated health maintenance organization in Central Texas, launched a pharmacistled medication management program (MMP) in 2006 for highrisk patients with diabetes. MMP patients received a diabetes copay waiver and met with ambulatory care pharmacists in the outpatient setting 4 to 12 times per year. The purpose of this study was to evaluate the 2-year impact of the MMP on glycemic control and costs for patients enrolled in the MMP.

\section{Methods}

\section{Study Design and Data Source}

This study utilized a retrospective, quasi-experimental study design that matched MMP patients to control patients in order to assess 2-year clinical and financial outcomes. An integrated health care system in Central Texas conducted the study from August 1, 2006, to August 31, 2012. For MMP patients, the index date was the date of enrollment into the MMP. The baseline period was defined as the year (365 days) before MMP enrollment. The follow-up period was 2 years after the index date. Control patients were matched to MMP patients if they met all inclusion criteria. For control patients, the index date was 1 day before any Alc reading greater than 7.5. The study was approved by Scott \& White Health Plan's Investigational Review Board (IRB) before initiation.

Patient-level data were extracted from pharmacy claims, medical claims, electronic medical records, and health plan enrollment data for MMP and control patients during the study period.

\section{Study Population}

Patients were enrolled on a "rolling basis" starting on August 1, 2006. Inclusion criteria were as follows: continuous enrollment throughout the study period; aged between 18 to 62 years on the index date; ability to communicate in English; confirmed diagnosis of type 2 diabetes at least 1 year before enrollment (or matched outpatient visit for control patients); baseline Alc $>7.5 \%$; continuous enrollment in the health plan for at least 3 years; and at least 1 hypoglycemic medication during the baseline and postenrollment period. Additionally, patients had to be enrolled in the MMP for at least 2 years. The average length of enrollment for MMP patients was 1,556 days, or 4.26 years. Diabetes diagnosis was confirmed using the definitions from the Healthcare Effectiveness Data and Information Set developed by the National Committee for Quality Assurance, which included the following International Classification of Diseases, Ninth Revision, Clinical Modification (ICD-9-CM) codes: 250.xx, 357.2x, 362.0x, and 366.41. ${ }^{15}$ Control patients were identified from claims data and met the same inclusion criteria; however, patients were excluded from the control group if the patients were previously enrolled in any other of the institution's MMPs.

Our study's exclusion criteria were the following: history of type 1 diabetes, enrollment in other nondiabetes MMPs, coverage by Medicare or Medicaid, or refusal from the patient's 
physician. After applying all inclusion and exclusion criteria to the MMP group (330 patients of whom 219 patients agreed to participate in the study) and standard care group (3,644 patients), 81 MMP patients and 1,433 standard care patients remained for matching. The final analysis included 75 patients in each group (matched 1:1) who met all the inclusion and exclusion criteria.

\section{Matching MMP Cohort to Standard Care Cohort}

The 2 cohorts were matched using the following criteria: gender, age \pm 5 years, Alc at baseline $\pm 0.5 \%$ within 1 year, insulin use, and physical comorbidity per quartile rank of the Selim Physical Comorbidity Index. Insulin use was determined by searching the patient's pharmacy claims for any insulin claims (AHFS code 68:20.08) during the 365-day period before the index date for the control and MMP group. Co-occurring conditions were captured using the Selim Physical Comorbidity Index, which sums 30 chronic medical conditions (range 0-30). ${ }^{16-18}$ Of 81 MMP patients, 75 were matched ( $7 \%$ unmatched) for a combined sample of 150 patients.

\section{MMP Intervention}

Pharmacists participating in the MMP worked under a collaborative agreement with physicians that allowed pharmacists to adjust diabetic medications when appropriate. Patients in the MMP group met with an ambulatory care pharmacist in a private patient counseling office located at 1 of the 12 Scott $\&$ White's retail outpatient pharmacies or clinics. During the first year, MMP patients had 1 appointment per month with an ambulatory care pharmacist. At the initial 60-minute appointment, the pharmacist evaluated the patient's therapy needs and developed a patient care plan, with the goal of achieving an Alc $<7 \%$, unless otherwise specified, per American Diabetes Association (ADA) recommendations. ${ }^{19}$ During each 30-minute follow-up appointment, the pharmacist spent approximately 20 minutes teaching the patient about different diabetes-related topics in this listed order: overview and goals of therapy, selfmonitoring blood glucose/appropriate glucometer use, acute complications, medication timing and administration, nutrition requirements, carbohydrate counting, exercise, medication education and adherence, chronic complications, foot care, psychosocial issues, and stress management. In the second year of program enrollment, pharmacists met with the patients once every 3 months or more often if necessary. The visit cost with the pharmacist led to a charge to the health plan of approximately $\$ 75$.

In addition, the pharmacist monitored the patient as recommended by the ADA, evaluated home blood glucose journals, and made medication adjustments, as allowed under a collaborative practice agreement with physicians. While flexibility existed in the program to address specific areas of concern for each patient at each visit, a general listing of all scheduled procedures for each appointment was specified in the protocol.
TABLE 1 Baseline Characteristics by Groups

\begin{tabular}{|c|c|c|c|c|}
\hline \multirow[b]{2}{*}{ Mean Alc, \% (SD) } & \multicolumn{2}{|c|}{$\begin{array}{l}\text { MMP } \\
\mathrm{n}=75\end{array}$} & \multicolumn{2}{|c|}{$\begin{array}{c}\text { Control } \\
\mathrm{n}=75\end{array}$} \\
\hline & 9.30 & (1.36) & 9.26 & $(1.32)$ \\
\hline Mean age, years (SD) & 53.00 & $(6.20)$ & 53.30 & $(6.10)$ \\
\hline $\begin{array}{l}\text { Mean Selim Physical } \\
\text { Comorbidity score (SD) }\end{array}$ & 3.32 & $(1.35)$ & 3.26 & (1.34) \\
\hline \multicolumn{5}{|l|}{ Gender, n (\%) } \\
\hline Female & 41 & $(54.7)$ & 41 & $(54.7)$ \\
\hline Male & 34 & $(45.3)$ & 34 & $(45.3)$ \\
\hline Insulin use & 42 & $(56.0)$ & 42 & $(56.0)$ \\
\hline
\end{tabular}

Alc = glycated hemoglobin A1c; MMP = medication management program; $S D=$ standard deviation.

Patients enrolled in the MMP received the following at no cost: visits with the pharmacist, medications for diabetes, and testing supplies for diabetes. At the first MMP appointment, patients were asked to sign a contract waiver specifying the terms under which prescription copayments were to be waived, as well as the list of approved medications and supplies. The contract required that the patient miss no more than 1 appointment (cancellations that were made ahead of time were allowed).

\section{Outcomes Analysis}

The primary outcome was the 2-year change in Alc between the MMP patients and the matched control patients. Secondary outcomes were MMP and control patients' costs in the first and second year from the health plan perspective, as well as Alc at year 1 and year 2. Baseline Alc reading was defined as the most recent reading within 6 months before the index date for MMP patients (matched Alc date for control). If no reading was available within 6 months before enrollment, then the first reading within 3 months after enrollment was counted as the baseline reading. The postperiod (2-year) Alc was the reading closest to the end of the follow-up period (within 6 months). Patients who did not have a follow-up Alc reading were excluded from the analysis.

We also evaluated medical and pharmacy claims for costs. Annual per patient health care costs were analyzed by group and type of service. ${ }^{20}$ Inpatient costs included all hospitalization events and claims occurring in the hospital setting. Outpatient costs included all claims occurring in a clinic, physician's office, or emergency department, as well as the MMP pharmacy locations (pharmacists billed for the MMP intervention). Pharmacy costs included all oral and injective drugs as well as testing supplies. Costs were adjusted to 2015 U.S. dollars assuming a 3\% annual inflation rate. Because this study was not powered to detect differences in costs, mean and median costs with standard deviations were reported. 
TABLE 2 Changes in Alc at Year 1 and Year 2

\begin{tabular}{|c|c|c|c|}
\hline & $\begin{array}{c}\text { MMP } \\
\mathbf{n}=75 \\
\text { Mean (SD) }\end{array}$ & $\begin{array}{c}\text { Control } \\
\mathbf{n}=75 \\
\text { Mean (SD) }\end{array}$ & $P$ Value \\
\hline Baseline Alc & $9.30 \quad(1.36)$ & $9.26 \quad(1.32)$ & $0.342^{a}$ \\
\hline l-year Alc & $(1.28)$ & $(1.56)$ & $0.012^{b}$ \\
\hline 2-year Alc & $(1.27)$ & $(1.80)$ & $0.014^{b}$ \\
\hline Change in Alc ( 2 years) & $(1.57)$ & $(1.75)$ & $0.009 \mathrm{~b}$ \\
\hline \multicolumn{4}{|c|}{$\begin{array}{l}\text { aWilcoxon signed-rank test. } \\
\text { bPaired t-test. } \\
\text { Alc=glycated hemoglobin Alc; MMP= medication management program; } \\
\text { SD= standard deviation. }\end{array}$} \\
\hline
\end{tabular}

Paired t-tests and Wilcoxon signed-rank tests were employed for assessing differences in the primary outcome between MMP patients and matched controls. Analyses were performed using SAS version 9.3 (SAS Institute, Cary, NC). For all statistical tests, an a priori significant level of $\alpha=0.05$ was assumed.

\section{Results}

The final analysis included 75 patients in each group (matched 1:1) who met all the inclusion and exclusion criteria. Among a sample of 150 patients ( $75 \mathrm{MMP}, 75$ control), $54.7 \%$ of patients were female, and $56.0 \%$ were insulin users (Table 1). Patients in the MMP and control groups had the following attributes at baseline: mean age, 53.0 years and 53.3 years, respectively; Selim Physical Comorbidity Score, 3.32 and 3.26, respectively; Alc, 9.30 and 9.26, respectively.

MMP patients had lower mean Alc readings compared with standard care patients at year $1(8.18 \%$ vs. $8.69 \% ; P=0.009)$ and year 2 (8.06\% vs. $8.67 \% ; P=0.012$; Table 2 ). The 2 -year average decrease in Alc was larger for the MMP patients compared with the control patients $(-1.24$ vs. $-0.59 ; P=0.014)$. The Alc difference-in-difference was 0.65 favoring the MMP patients.

During the year before the index date, the median costs associated with MMP patients and standard care patients were $\$ 3,162$ and $\$ 2,174$, respectively, for medical care (inpatient, outpatient, and emergency room) and $\$ 3,025$ and $\$ 2,494$ for pharmaceutical products (drugs and testing supplies). During the second year after the index date, the median costs associated with MMP patients and standard care patients were $\$ 4,687$ and $\$ 5,262$, respectively, for medical care and $\$ 5,574$ and $\$ 3,211$, respectively, for pharmaceutical products. The difference (median) in medical costs between the second year and the baseline period were $\$ 1,525$ for the MMP patients and $\$ 3,088$ for standard care patients. The difference (median) in pharmacy costs between the second year and the baseline period were $\$ 2,549$ and $\$ 717$ for standard care patients (Table 3).

\section{Discussion}

The impact of the MMP and copay waiver on reducing Alc over a 2-year period trended as expected. The decrease in Alc for control patients was significantly lower than for MMP patients. Additionally, the change in Alc for control patients did not change from baseline to the second year (Table 2). Control patients received standard medical and pharmacological care, while the MMP patients received standard care plus pharmacist-led medication management and education. This study was not set up to examine the isolated impact of the copayment waiver or the pharmacist-led MMP; therefore, we cannot conclude the effect of either independently.

Although the study was not powered to detect differences in costs, the second-year increase in medical costs versus the baseline period was numerically lower for MMP patients compared with control patients. MMP patients, however, had numerically higher pharmacy costs versus control patients. In addition, current literature on the financial outcomes of pharmacist interventions in patients with diabetes suggest that a longer time period of at least 3 to 5 years is necessary to obtain a financial benefit from pharmacist-led outpatient diabetes management. ${ }^{12,13}$ Diabetes is a chronic disease affecting patients over a long period of time, with ailments not limited to costly macrovascular and microvascular complications. The significant reduction in Alc for MMP patients starting in the

TABLE 3 Mean and Median Annual Costs Per Patient from the Health Plan Perspective (2015 Dollars)

\begin{tabular}{|c|c|c|c|c|c|c|c|c|}
\hline & $\begin{array}{c}\text { MMP } \\
\text { Median } \\
\text { Medical } \\
\text { Cost }^{a}\end{array}$ & $\begin{array}{c}\text { MMP } \\
\text { Mean (SD) } \\
\text { Medical } \\
\text { Cost }^{\mathrm{a}}\end{array}$ & $\begin{array}{c}\text { MMP } \\
\text { Median } \\
\text { Pharmacy } \\
\text { Cost }\end{array}$ & $\begin{array}{c}\text { MMP } \\
\text { Mean (SD) } \\
\text { Pharmacy } \\
\text { Cost }\end{array}$ & $\begin{array}{l}\text { Control } \\
\text { Median } \\
\text { Medical } \\
\text { Cost }^{\mathrm{a}}\end{array}$ & $\begin{array}{c}\text { Control } \\
\text { Mean (SD) } \\
\text { Medical } \\
\text { Cost }^{\mathrm{a}}\end{array}$ & $\begin{array}{c}\text { Control } \\
\text { Median } \\
\text { Pharmacy } \\
\text { Cost }\end{array}$ & $\begin{array}{c}\text { Control } \\
\text { Mean (SD) } \\
\text { Pharmacy } \\
\text { Cost }\end{array}$ \\
\hline Baseline period ${ }^{b}$ & $\$ 3,162$ & $\$ 6,987(10,163)$ & $\$ 3,025$ & $\$ 4,255 \quad(4,877)$ & $\$ 2,174$ & $\$ 5,629 \quad(9,738)$ & $\$ 2,494$ & $\$ 3,236 \quad(2,713)$ \\
\hline First yearc & $\$ 4,383$ & $\$ 7,732 \quad(7,838)$ & $\$ 4,780$ & $\$ 6,501 \quad(6,123)$ & $\$ 3,311$ & $\$ 6,576 \quad(9,172)$ & $\$ 2,746$ & $\$ 4,011 \quad(3,502)$ \\
\hline Second yeard & $\$ 4,687$ & $\$ 9,541(12,798)$ & $\$ 5,574$ & $\$ 7,626 \quad(7,012)$ & $\$ 5,262$ & $\$ 9,414(12,082)$ & $\$ 3,211$ & $\$ 4,023 \quad(3,461)$ \\
\hline
\end{tabular}

a Medical cost=inpatient, outpatient, and emergency department costs.

bBaseline period $=365$ days before the index date.

cFirst year $=365$ days after the index date.

dSecond year $=366$ to 730 days after the index date.

$M M P=$ medication management program; SD = standard deviation. 
first year and sustained into the second year is indicative of the effectiveness of the pharmacist intervention combined with the diabetes copay waiver.

When compared with the baseline period, medical cost increases for MMP were lower compared with control patients in the second year. For MMP patients, outpatient costs included a charge of approximately $\$ 75$ per visit, which was billed to their insurance.

Pharmacy costs were numerically greater for MMP patients in the second year compared with the baseline period. Possible drivers for increased drug costs for MMP patients are greater use of branded medications, which will be examined to optimize the program. At the time of the study, the copay waiver covered all diabetes drugs regardless of formulary status. It is possible that the knowledge of the copay waiver led to more liberal prescribing of higher cost brand medications by prescribers. In the future, we will consider covering only formulary drugs, while giving patients the option of a cost-share for nonformulary agents.

\section{Limitations}

Several limitations to this study should be noted. Although group equivalency was enhanced by matching on key characteristics, including age, gender, baseline Alc, and Selim Physical Comorbidity Index, there may be unobservable behavioral differences between groups. MMP patients may be more active in seeking medical care or have higher disease burden as indicated by higher baseline medical costs in the baseline period. At the same time, it is possible that the MMP patients are more willing to change their health behavior, since they are required to meet with an ambulatory care pharmacist at regular intervals to stay in the program. This study examined the change in Alc for patients receiving the pharmacist intervention and the copay waiver versus control patients; therefore, the extent of the impact of the pharmacist intervention, copay waiver, or patient behaviors individually is unknown. In addition, testing may be an issue because some patients were tested for Alc using a blood draw at baseline but were then tested with the finger-prick method 1 year after enrollment or vice versa. However, this inconsistency in testing methods may occur to the same extent in both groups. The use of prescription claims and medical claims have their limitations in painting a clear clinical picture for patients, since they are primarily intended for administrative and billing purposes. Finally, because of the long-term sequelae associated with diabetes, the 2-year follow-up period may not provide adequate insight into the effectiveness of the MMP. The investigators of this study plan on examining outcomes over a longer period of time.

\section{Conclusions}

A pharmacist-led diabetes MMP, combined with a diabetes drug copay waiver, was effective in significantly reducing Alc over a 2-year period for type 2 diabetic patients in this regional health plan. In addition to greater Alc control, MMP patients had numerically lower inpatient costs than standard care patients, while outpatient costs were similar. Overall drug use and expenditure were significantly higher for MMP patients. Further research is needed to examine the impact of the MMP on long-term disease progression, reduction of diabetes-related complications, medication adherence, and quality of life.

\section{Authors}

JOHN J. KO, PharmD, MS, is Associate Director, Health Economics and Outcomes Research, Novartis, East Hanover, New Jersey; KANGHO SUH, PharmD, MS, is PhD Candidate, Pharmaceuticals Outcomes Research \& Policy Program, University of Washington, Seattle; EILEEN M. STOCK, PhD, is Research Statistician, Center for Applied Health Research, jointly sponsored by Central Texas Veterans Health Care System and Baylor Scott $\&$ White Health, Temple, Texas; YOONA KIM, PharmD, is Researcher, Health Economics, Proteus Digital Health, Redwood City, California; JACKIE LU, PharmD, MS, is Regional Clinical Account Director, Medical Affairs, AstraZeneca, New York City, New York; and KAREN RASCATI, PhD, is Professor of Pharmacy, Health Outcomes and Pharmacy Practice, College of Pharmacy, The University of Texas at Austin. JOYCE JUAN, PharmD, is Clinical Pharmacist, CDM Pharmacy; PATRICIA A. TABOR, PharmD, is Manager, Clinical Pharmacy Services; and PAUL J. GODLEY, PharmD, is Director, Health Plan Pharmacy Services, Scott \& White Health Plan, Temple, Texas.

AUTHOR CORRESPONDENCE: Paul J. Godley, PharmD, Health Plan Pharmacy Services, Scott \& White Health Plan, 1206 W. Campus Dr., Temple, TX 76502. Tel.: 254.298.6123; Fax: 254.298.6189; E-mail: pgodley@sw.org.

\section{DISCLOSURES}

Scott $\&$ White Health Plan supported this research by funding the copay waivers used in this study. Ko was a fellow at Scott \& White Health Plan during year 1 of this study and a fellow at Novartis Pharmaceuticals during year 2 of this study. Novartis Pharmaceuticals did not have any input in this study nor did it contribute any funding or support for this research. Ko, Lu, Suh, and Kim were paid fellows at Scott \& White Health Plan at the time of this study. The authors declare no other potential conflicts of interest.

Study design was created by Godley, Kim, and Ko, with assistance from Lu, Stock, and Suh. Ko, Juan, Suh, and Tabor collected the data, with data interpretation performed by Stock, Lu, and Rascati. The manuscript was written by Ko, Lu, Stock, and Kim and revised by Ko, Lu, Rascati, and Godley.

\section{REFERENCES}

1. Dall TM, Zhang Y, Chen YJ, Quick WW, Yang WG, Fogli J. The economic burden of diabetes. Health Aff (Millwood). 2010;29(2):297-303.

2. Kim S. Burden of hospitalizations primarily due to uncontrolled diabetes: implications of inadequate primary health care in the United States. Diabetes Care. 2007;30(5):1281-82. 
3. Li R, Zhang P, Barker LE, Chowdhury FM, Zhang X. Cost-effectiveness of interventions to prevent and control diabetes mellitus: a systematic review. Diabetes Care. 2010;33(8):1872-894

4. Machado M, Bajcar J, Guzzo GC, Einarson TR. Sensitivity of patient outcomes to pharmacist interventions. Part I: systematic review and metaanalysis in diabetes management. Ann Pharmacother. 2007;41(10):1569-82

5. Smith M. Pharmacists' role in improving diabetes medication management. J Diabetes Sci Technol. 2009;3(1):175-79.

6. Cranor CW, Bunting BA, Christensen DB. The Asheville Project: longterm clinical and economic outcomes of a community pharmacy diabetes care program. J Am Pharm Assoc (2003). 2003;43(2):173-84.

7. Fera T, Bluml BM, Ellis WM. Diabetes Ten City Challenge: final economic and clinical results. J Am Pharm Assoc (2003). 2009;49(3):383-91.

8. Wubben DP, Vivian EM. Effects of pharmacist outpatient interventions on adults with diabetes mellitus: a systematic review. Pharmacotherapy. 2008:28(4):421-36

9. Wagner EH, Sandhu N, Newton KM, McCulloch DK, Ramsey SD, Grothaus LC. Effect of improved glycemic control on health care costs and utilization. JAMA. 2001;285(2):182-89.

10. Sease JM, Franklin MA, Gerrald KR. Pharmacist management of patients with diabetes mellitus enrolled in a rural free clinic. Am J Health Syst Pharm. 2013;70(1):43-47

11. Ip EJ, Shah BM, Yu J, Chan J, Nguyen LT, Bhatt DC. Enhancing diabetes care by adding a pharmacist to the primary care team. Am J Health Syst Pharm. 2013:70(10):877-86

12. Juarez DT, Goo R, Tokumaru S, Sentell T, Davis JW, Mau MLM Association between sustained glycated hemoglobin control and healthcare costs. Am J Pharm Benefits. 2013;5(2):59-64. Available at: http://www.ajmc.com/ publications/ajpb/2013/AJPB_MarApr2013/Association-Between-SustainedHemoglobin-Control-and-Healthcare-Costs. Accessed November 5, 2015.
13. Yu J, Shah BM, Ip EJ, Chan J. A Markov model of the cost-effectiveness of pharmacist care for diabetes in prevention of cardiovascular diseases: evidence from Kaiser Permanente Northern California. J Manag Care Pharm. 2013;19(2):102-14. Available at: http://www.amcp.org/JMCP/2013/ March/16230/1033.html

14. Nair KV, Miller K, Park J, Allen RR, Saseen, JJ, Biddle V. Prescription co-pay reduction program for diabetic employees. Popul Health Manag. 2010;13(5):235-45.

15. National Committee for Quality Assurance. HEDIS 2009 Volume 2: Technical Update. Available at: http://www.ncqa.org/Programs/ Accreditation/PolicyUpdatesSupportingDocuments/HEDIS TechnicalUpdates.aspx. Accessed November 5, 2015

16. Pugh MJ, Copeland LA, Zeber JE, et al. The impact of epilepsy on health status among younger and older adults. Epilepsia. 2005;46(11):1820-27.

17. Copeland LA, Zeber JE, Wang CP, et al. Patterns of primary care and mortality among patients with schizophrenia or diabetes: a cluster analysis approach to the retrospective study of healthcare utilization. BMC Health Serv Res. 2009;9:127.

18. Selim AJ, Fincke G, Ren XS, et al. Comorbidity assessments based on patient report: results from the Veterans Health Study. J Ambul Care Manage. 2004; 27(3):281-95

19. American Diabetes Association. Standards of medical care in diabetes-2012. Diabetes Care. 2012;35(Suppl 1):S11-S63.

20. Epidemiology of Diabetes Interventions and complications (EDIC) Research Group. Epidemiology of Diabetes Interventions and Complications (EDIC): design, implementation, and preliminary results of a long-term follow-up of the Diabetes Control and Complications Trial cohort. Diabetes Care. 1999;22(1):99-111. 